\title{
VARIABLE OBJECTS IN A COMPLETE SAMPLE OF FAINT RADIO SOURCES
}

\author{
D.R. Altschuler ${ }^{1,2}$ and T. Forkert ${ }^{1}$ \\ ${ }^{1}$ Max-Planck-Institut für Radioastronomie \\ Auf dem Hüge1 69, D-5300 Bonn 1, F.R.G. \\ ${ }^{2}$ Physics Department, Faculty of Natural Sciences, \\ University of Puerto. Rico, Rio Piedras, P.R. 00931, U.S.A.
}

ABSTRACT. Measurements of the $5 \mathrm{GHz}$ flux densities of faint radio sources ( $\sim 80 \mathrm{mJy}$ ) in a complete sample have been made at three epochs spanning a 15-year timebase. These measurements provide data for a statistical investigation of activity in faint radio sources. Here we report the results for some outstanding objects.

\section{INTRODUCTION}

Since the discovery of variability in the flux density of radio sources by Dent (1965) and Sholomitskii (1965), the study of their properties has been the topic of a large amount of work. Not surprisingly most studies of active radio sources (ARS) have concentrated on relatively strong sources ( $S>1 \mathrm{Jy}$ ) often selected because of their known or suspected activity (Altschuler and Wardle 1977, Andrew et a1. 1978, A1ler et a1. 1985).

While this type of work provides input to the physics relevant to the ARS, and allows confrontations with specific models, it provides little information on the causative agents for this activity. An answer to this question might be possible through the study of complete flux density limited samples, and the search for statistical relations between variability and other parameters which can be determined for the samples. The study of complete samples of faint sources might also show the effects of evolution and population changes. The difference in the mean spectra between strong ( $\mathrm{S}>1.5 \mathrm{Jy}$ ) and weak sources ( $>15 \mathrm{mJy}$ ) (Pauliny-Toth et a1. 1974, Condon and Ledden 1981) suggests a difference in their physical properties, although the identification content of strong and faint samples at $5 \mathrm{GHz}$ are similar (Condon et al. 1975).

In order to pursue these questions a $5 \mathrm{GHz}$ survey of a strip of extragalactic sky was carried out in 1981, covering part of the region surveyed by the NRAO survey of faint sources 10 years earlier (Davis 1971). Both surveys were carried out with the NRAO 91-meter telescope, the first one detecting 254 sources stronger than $41 \mathrm{mJy}$, and the second 
one detecting 882 sources stronger than $15 \mathrm{mJy}$. Results of this second survey are reported by Altschuler (1986).

To improve the available data base we have undertaken measurements of third epoch flux densities and spectral indices between 2.7 and $4.75 \mathrm{GHz}$ of al1 sources detected above $50 \mathrm{mJy}$ in the 1981 survey (210 sources), or sources below this limit but detected in the 1971 survey (30 sources). The measurements were carried out with the 100-meter radio telescope of the Max-Planck-Institut für Radioastronomie, located in Effelsberg, F.R.G., on 8-10 March 1986 at $4.75 \mathrm{GHz}$ and on 28 February and 14-17 March 1986 at 2.7 GHz (Forkert and Altschuler 1986). The three sets of measurements cover a total time span of 15 years (1971.0, 1981.9, 1986.2) and provide data for a study of the variability occurring in a complete sample of faint radio sources. The median flux density of these sources is about $80 \mathrm{mJy}$.

\section{RESULTS}

Here we provide information on some sources which are at the extreme of the variability amplitude distribution when the three epoch flux densities are compared after a careful adjustment of the flux density scales correcting for the slightly different observing frequencies and for small systematic changes in the calibration.

Table I gives the data for these sources, several of which have changed their flux density by a factor larger than two. The $2.7 \mathrm{GHz}$ data when compared with earlier measurement by Pauliny-Toth et a1. (1972), shows the same trend as the $4.76 \mathrm{GHz}$ data. The spectral indices between the two frequencies are typical for variable sources. Figure 1 shows the flux density as a function of time for these sources, where some additional points from the literature have been included.

It is noteworthy that the flux density of most of these sources has changed systematically over the 15 year timebase. Such behaviour, although not the subject of much attention, is not uncommon in ARS. A good example is 2134+004 (Andrew et al. 1978), which over ten years decreased its flux density by a factor of 2 , but does not show large short-term variations.

Because of the sparse sampling, our program will determine for these sources a larger overall change than for sources which vary on a shorter time-scale about some constant average value, so that no significance should be attributed to the fact that they have shown the largest variations in our sample. A discussion of the statistical variability found in this complete sample is given by Altschuler and Forkert (1986).

This research was supported by a grant from the division of international programs of the NSF (INT-8510665).

\section{REFERENCES}

Aller, H.D., Aller, M.F., Latimer, E., Hodge, P.E.: 1985, Astrophys. J. Supp1. $\underline{\underline{59}}, 513$ 
Andrew, B.H., MacLeod, J.M., Harvey, J.A., Medd, W.J.: 1978, Astron. J. 83,863

Altschuler, D.R. and Wardle, J.F.C.: 1977, Monthly Notices Roy. Astron. Soc. 179,153

Altschuler, D.R.: 1986, Astron. Astrophys. Suppl. (in press)

Altschuler, D.R. and Forkert, T.: 1986, (in preparation)

Condon, J.J., Balonek, T.J., Jauncey, D.L.: 1975, Astron. J. 80, 887

Condon, J.J. and Ledden, J.E.: 1981, Astron. J. 86, 643

Davis, M.M.: 1971, Astron. J. Zㅡㅡ, 980

Dent, W.A.: 1965, Science $1 \underline{\underline{4}} \underline{\underline{\underline{8}}}, 1458$

Fanti, R., Ficarra, A., Formiggini, L., Gioia, I., Padriel1i, L.: 1974, Astron. Astrophys. 32, 155

Forkert, T. and Altschuler, D.R.: 1986, (in preparation)

Machalski, J. and Condon, J.J.: 1979, Astron. J. 84, 164

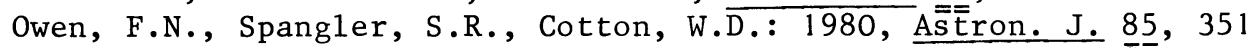

Pauliny-Toth, I.I.K., Kellermann, K.I., Davis, M.M., Fomaloñ $\overline{\bar{t}}$, E.B., Shaffer, D.B.: 1972, Astron. J. 77, 265

Pauliny-Toth, I.I.K., Witzel, A., Preuss, E.: 1974, Astron. Astrophys. 35,421

Shoİomitskii, G.B.: 1965, Sov. Astron. $\stackrel{9}{=} 516$

TABLE I. Outstanding Objects

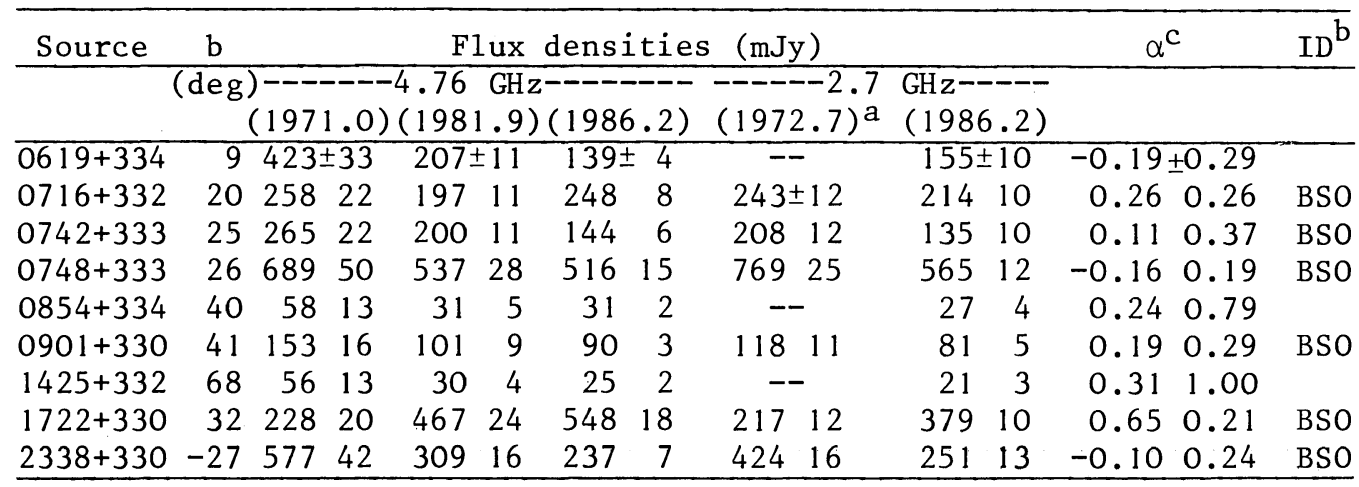

a) Flux densities from Pauliny-Toth et a1. (1972)

b) Identifications from Condon et al. (1975) and Fanti et a1. (1974) BSO = blue stellar object

c) Spectral index: $s \propto v^{\alpha}$ 


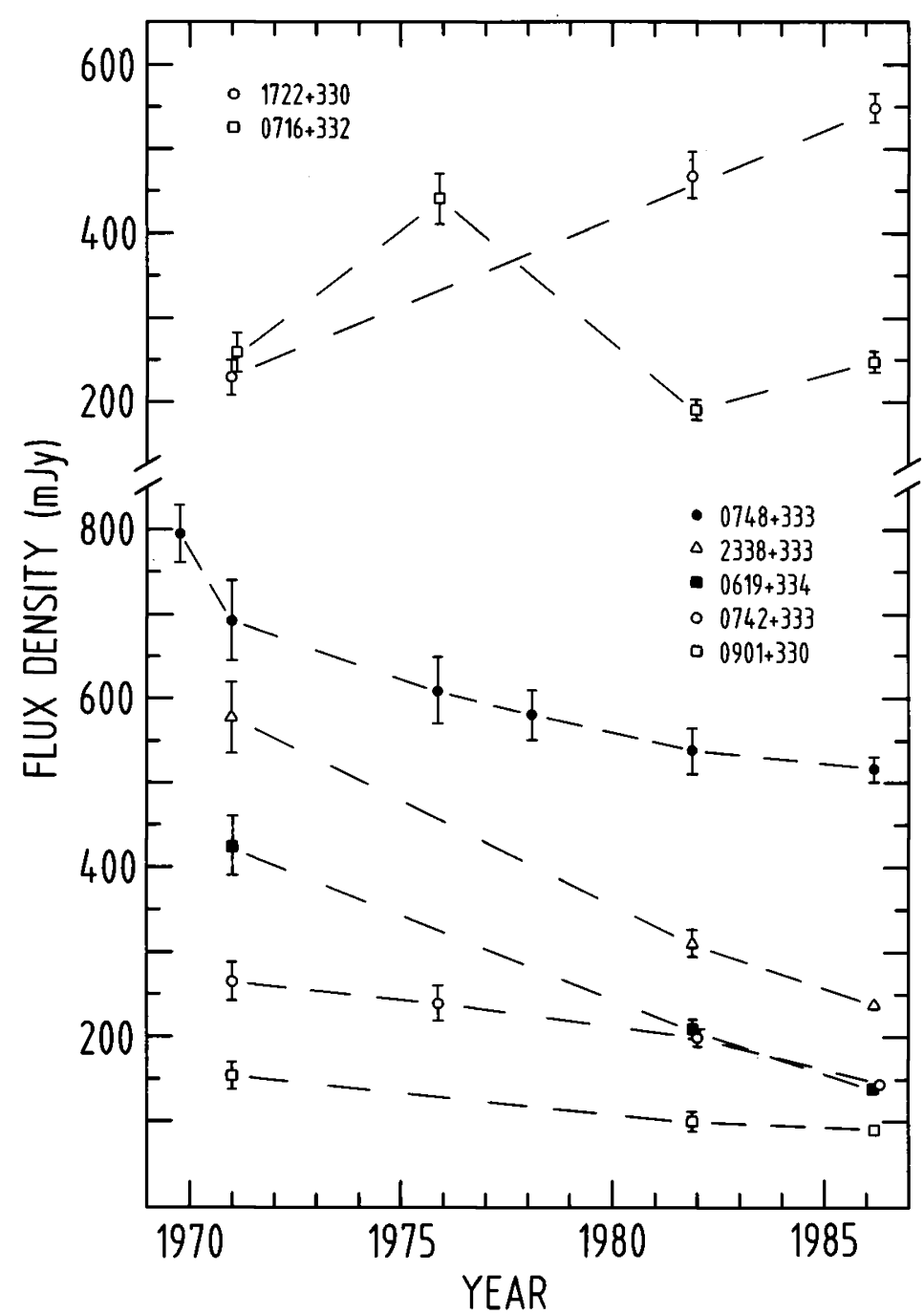

Figure 1. Flux density as a function of time for some outstanding sources. Additional data is from the following:

1969.6 Pauliny-Toth et a1. (1972)

1975.9 Machalski and Condon (1979)

1978.1 Owen et 1a. (1980) 